Revista Agrarian

ISSN: 1984-2538

\title{
Características biológicas de parasitoides de ovos criados em diferentes hospedeiros
}

\section{Biological characteristics of egg parasitoid reared on different hosts}

\section{Ariana Lisboa Meira ${ }^{1 *}$, Gustavo Martins Strum², Fernando Domingo Zinger ${ }^{3}$, Leonardo Mardgan², Dirceu Pratissoli ${ }^{2}$}

\author{
${ }^{(1)}$ Universidade Estadual do Sudoeste da Bahia, Vitória da Conquista, BA. \\ ${ }^{(2)}$ Universidade Federal do Espírito Santo (UFES), Centro de Ciências Agrárias (CCA) \\ (3) Instituto Federal de Educação, Ciência e Tecnologia (IF), Campus Lages, R. Heitor Villa Lobos, 222 - São \\ Francisco, Lages - SC, 88506-400 *arilismeira@yahoo.com.br
}

Recebido em: 29/04/2015

Aceito em:24/04/2016

\begin{abstract}
Resumo: As espécies de Trichogramma são agentes biológicos que contribuem para o controle de lepidópteros. Objetivou-se neste trabalho identificar qual espécie de Trichogramma apresenta melhor desempenho biológico quando criados em ovos de Anagasta kuehniella Zeller (Lepidoptera: Pyralidae), Helicoverpa zea (Boddie) (Lepidoptera: Noctuidae) e Plutella xylostella Linnaeus (Lepidoptera: Plutellidae). Desta forma os parâmetros biológicos avaliados foram período de duração de ovo a adulto e percentual de emergência e no estágio adulto avaliou-se número de ovos parasitados, número de indivíduos por ovo e razão sexual em Trichogramma atopovirilia Oatman \& Planter (Hymenoptera: Trichogrammatidae) e Trichogramma exiguum Pinto \& Planter (Hymenoptera: Trichogrammatidae). Para a duração do ciclo de desenvolvimento, A. kuehniella, H. zea e P. xylostella não apresentaram diferença quando criados com as mesmas espécies de Trichogramma e não houve diferença para o percentual de emergência entre os três hospedeiros em estudo. Em relação aos parâmetros avaliados no estágio adulto, para número de ovos parasitados, somente $A$. kuehniella, diferenciou entre as espécies de Trichogramma. O número de indivíduos por ovo, os maiores valores foram observados quando as espécies de Trichogramma foram criadas com ovos de H. zea. A razão sexual, T. atopovirilia diferiu apenas para A. kuehniella. As duas espécies de Trichogramma estão aptas a se desenvolverem nos hospedeiros A. kuehniella e $P$. xylostella. Contudo para o hospedeiro $H$. zea, T. exiguum foi à espécie que obteve melhor desempenho, parasitando maior número de ovos e obtendo maior número de indivíduos por ovo.
\end{abstract}

Palavras-chave: hospedeiro alternativo, lagarta da espiga, traças das crucíferas, Trichogramma

Abstract. The Trichogramma species are biological agents that contribute to the control of Lepidoptera. The aim of this work was to identify what species of Trichogramma has better biological performance when created in eggs of Anagasta kuehniella Zeller (Lepidoptera: Pyralidae), Helicoverpa zea (Boddie) (Lepidoptera: Noctuidae) and Plutella xylostella Linnaeus (Lepidoptera: Plutellidae). Thus the biological parameters evaluated were egg duration of period from egg to adult and emergency percentage and adult stage evaluated the number of parasitized eggs, number of individuals per egg and sex ratio in Trichogramma atopovirilia Planter \& Oatman (Hymenoptera: Trichogrammatidae) and Trichogramma exiguum Pinto \& Planter (Hymenoptera: Trichogrammatidae). For the period from egg to adult, A. kuehniella, H. zea and P. xylostella, showed no difference when created with the species of Trichogramma for the duration of the development cycle and no difference for the emergency percentage among the three hosts study. In relation to the parameters evaluated in the adult stage, for number of parasitized eggs, only A. kuehniella differed between species of Trichogramma. The number of individuals per egg, the highest values was observed when the Trichogramma species were created with $H$. zea eggs. The sex ratio, T. atopovirilia differed only for A. kuehniella. The two species of Trichogramma are apt to develop in host A. kuehniella and P. xylostella. However to the host $H$. zea, T. exiguum was the species that had the best performance, parasitizing more eggs

Key words: alternative hosts, corn earworm, diamondback moth, Trichogramma

Meira et al., v.10, n.35, p. 10-17, Dourados, 2017 


\section{잉 \\ Revista Agrarian \\ ISSN: 1984-2538}

\section{Introdução}

O repolho, Brassica oleracea var. capitata L., é produzido em regiões temperadas a subtropicais. A cultura depara com problemas, dentre eles, a ocorrência de pragas, podendo ocasionar prejuízos consideráveis na produção. Entre as pragas que atacam as brássicas, destaca-se a traça-das-crucíferas, Plutella xylostella L. (Lepidoptera: Plutellidae). As lagartas jovens causam prejuízo econômico, pois raspam o tecido foliar, e posteriormente surgem furos que dependendo da severidade pode causar até a morte das plantas (Cardoso et al., 2012). Outro fator importante que torna este microlepidóptero prejudicial é o alto potencial biótico, pois seu ciclo de vida completa em16 dias e cada fêmea deposita aproximadamente 140 ovos (Boiça Júnior et al., 2011).

Outra espécie importante é a lagarta da espiga, Helicoverpa zea Boddie (Lepidoptera: Noctuidae), por ser um inseto polífago, causam danos em diversas culturas, entre elas, o milho e outras gramíneas, solanáceas, leguminosas, frutíferas e hortaliças. Em milho, os danos diretos podem chegar a $8 \%$, causando, ainda, danos indiretos através da abertura da espiga, permitindo a entrada de outras pragas, além de fungos que causam podridão e umidade, impossibilitando a comercialização da espiga (Freitas et al., 2010).

Estas pragas danificam áreas comerciais de crucíferas e milho, respectivamente, muitas vezes tornando as culturas impróprias para a comercialização e o consumo. O controle delas é feito principalmente com inseticidas. No entanto estes têm demonstrado baixa eficiência, em virtude das aplicações não serem realizadas no período correto e o hábito do inseto de se manter no interior das espigas e folhas, ficando protegido da calda inseticida (Foresti et al., 2012).

As aplicações intensivas tornam as populações resistentes e dificulta o manejo fitossanitário de pragas (Wang et al., 2010) aumentando o custo da produção, causando efeitos antagônicos ao agroecossistema, deixando os resíduos nos alimentos, além de eliminar os inimigos naturais (Boiça Júnior et al., 2013). É necessário buscar alternativas eficientes e ecologicamente compatíveis no controle destas pragas. Uma alternativa para a redução do uso de inseticidas é o emprego de agentes de controle biológico.
Dentre os agentes de controle biológico que atua sobre $P$. xylostella e $H$. zea, os parasitoides do gênero Trichogramma apresentamse como alternativa muito promissora, pois são microhimenópteros parasitóides de ovos, que atacam inúmeras espécies de pragas agrícolas e florestais, principalmente da ordem Lepidoptera (Vianna et al., 2011). Além destas pragas, as espécies de Trichogramma apresentam bons desempenhos biológicos quando criadas em ovos de Anagasta kuehniella Zeller (Lepidoptera: Pyralidae) que é um dos hospedeiros alternativos de criação em laboratório.

Os relatos de pesquisas, no Brasil, mencionando aspectos biológicos de espécies de Trichogramma parasitando ovos de P. xylostella e H. zea são escassos (Pereira et al., 2007; Pratissoli et al., 2008; Meira et al., 2011). Neste contexto objetivou-se neste trabalho identificar qual espécie de Trichogramma apresenta melhor desempenho biológico quando criados em ovos de P.xylostella, H. zea e A. kuehniella.

\section{Material e Métodos}

Local do experimento

$\mathrm{O}$ experimento foi conduzido no Laboratório de Entomologia do Núcleo de Desenvolvimento Científico e Tecnológico em Manejo Fitossanitário (NUDEMAFI) do Centro de Ciências Agrárias da Universidade Federal do Espírito Santo (CCA/UFES), Alegre, ES. O local

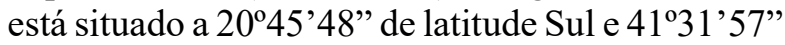
de longitude Oeste, e uma altitude aproximada de $250 \mathrm{~m}$.

\section{Criação do hospedeiro alternativo A. kuehniella}

Este hospedeiro foi criado em dieta contendo $60 \%$ de farelo de trigo, $37 \%$ de fubá de milho e $3 \%$ de levedura de cerveja. Os componentes da dieta foram homogeneizados e distribuídos em caixas plásticas $(30 \times 25 \times 10 \mathrm{~cm})$. Em cada caixa foram adicionados $0,4 \mathrm{~g}$ de ovos de A. kuehniella sobre a dieta, cobrindo-as com sacolas plásticas. Estas caixas foram mantidas em sala climatizada a $25 \pm 1{ }^{\circ} \mathrm{C}, 70 \pm 10 \%$ UR e $12 \mathrm{~h}$ de fotofase. Os adultos emergidos foram coletados diariamente com auxílio de aspirador de pó, sendo posteriormente transferidas para potes plásticos (20 x $25 \mathrm{~cm}$ ), contendo no seu interior tiras de tela de "nylon" dobradas em zig-zag, para facilitar a 


\section{- \\ Revista Agrarian}

ISSN: 1984-2538

oviposição. A parte superior dos potes foi vedada com tecido "voil" para evitar a fuga das mariposas.

\section{Criação e manutenção de T. atopovirilia e T. exiguum}

As espécies Trichogramma atopovirilia Oatman \& Planter e Trichogramma exiguum Pinto \& Planter (Hymenoptera: Trichogrammatidae) utilizadas neste estudo foram proveniente da criação estoque do Laboratório de Entomologia do CCA/UFES, coletadas em ovos de $H$. zea em plantios de milho do Centro Nacional de Pesquisa de Milho e Sorgo, Sete Lagoas, MG e em plantio de tomate em Muniz Freire, ES, respectivamente.

Para a manutenção das espécies de Trichogramma foram oferecidos ovos de $A$. kuehniella colados em retângulos de cartolina azul $(8 \times 2 \mathrm{~cm})$ com goma arábica diluída em água a $30 \%$. Esses ovos foram previamente inviabilizados em lâmpada germicida durante 50 minutos. Essas cartelas foram inseridas em recipientes de vidro $(8,5 \times 2,4 \mathrm{~cm})$, contendo adultos recém-emergidos das espécies T. atopovirilia e T. exiguum. Após $24 \mathrm{~h}$ de parasitismo, essas cartelas com os ovos parasitados foram armazenadas em sala climatizada a $25 \pm 1{ }^{\circ} \mathrm{C}, 70 \pm 10 \%$ UR e $12 \mathrm{~h}$ de fotofase.

\section{Criação da lagarta da espiga $H$. zea}

A coleta de $H$. zea foi realizada em plantios de milho, localizada na Fazenda Fortaleza, Alegre, ES e a criação foi desenvolvida em sala climatizada a $25 \pm 1{ }^{\circ} \mathrm{C}, 70 \pm 10 \%$ UR e $12 \mathrm{~h}$ de fotofase . Os adultos de $\mathrm{H}$. zea foram mantidos em gaiolas de PVC $(20 \times 25 \mathrm{~cm})$ revestidos internamente com folha de papel branco e as extremidades fechadas com tecido do tipo "voil", sendo oferecida diariamente uma solução de mel a $10 \%$ como substrato alimentar. Os ovos de $H$. zea foram coletados e acondicionados em recipientes plásticos, após a eclosão as lagartas foram individualizadas em tubos de vidro $(8,5 \times 2,5 \mathrm{~cm})$ preenchidos em até $1 / 4$ de seu volume com dieta artificial a base de feijão, germe de trigo e farelo de soja. Após o resfriamento da dieta as lagartas foram transferidas para o tubo, sendo mantidas nestes recipientes até o período de pupa. Posteriormente estes recipientes foram acondicionados em sala climatizada a $25 \pm 1{ }^{\circ} \mathrm{C}, 70 \pm 10 \%$ UR e $12 \mathrm{~h}$ de fotofase.

\section{Criação das traças das crucíferas P. xylostella}

As lagartas foram coletadas em plantios comerciais de repolho, em Vargem Alta, ES. Este inseto foi criado em gaiolas plásticas circulares transparentes, com uma abertura retangular lateral, vedada com tela de "nylon", para circular o ar. A parte superior da gaiola tinha um orifício onde foi colocada uma esponja embebida com solução de mel a $10 \%$, presa com uma rolha de pano fechando o orifício. Foram colocados discos de $8 \mathrm{~cm}$ de diâmetro de folhas de couve, Brassica oleracea var. acephala, sobre um copo plástico contendo papel filtro umedecido em água destilada, onde as fêmeas realizavam as posturas. Os discos foram trocados diariamente e acondicionados em placas de Petri até a eclosão das lagartas.

As lagartas recém-eclodidas foram posteriormente transferidas para recipientes plásticos $(15 \mathrm{~cm} \times 10 \mathrm{~cm} \times 5 \mathrm{~cm})$, alimentadas com folhas de couve previamente lavadas em água corrente. Estas foram trocadas diariamente até que todos os insetos atingissem a fase de pupa. As pupas foram coletadas e acondicionadas em tubos de vidro para obtenção dos adultos. Esta criação foi mantida em sala climatizada a $25 \pm 1^{\circ} \mathrm{C}, 70 \pm 10 \%$ UR e $12 \mathrm{~h}$ de fotofase.

\section{Biologia}

Para cada espécie de Trichogramma foram individualizadas quatro fêmeas recém-emergidas em tubos de vidro $(13 \times 1 \mathrm{~cm})$, alimentadas com mel puro, depositado na parede interna dos tubos com o auxílio de um estilete e tampados com filme plástico de PVC, mantidos em sala climatizada a 25 $\pm 1{ }^{\circ} \mathrm{C}, 70 \pm 10 \%$ UR e $12 \mathrm{~h}$ de fotofase. Para as fêmeas de cada espécie, individualizadas em tubos, foram oferecidos 20 ovos de A. kuehniella, H. zea e P. xylostella, separadamente, com até $24 \mathrm{~h}$ de desenvolvimento embrionário, colados em retângulos de cartolina de cor azul $(3 \times 0,4 \mathrm{~cm}) \mathrm{com}$ goma arábica diluída em água a $30 \%$. Sendo que os ovos de A. kuehniella e H. zea foram previamente inviabilizados em lâmpada germicida durante $50 \mathrm{e}$ 30 minutos, respectivamente. Após $5 \mathrm{~h}$ de exposição das fêmeas de Trichogramma em ovos de A. kuehniella, $H$. zea e $P$. xylostella, foram retiradas dos tubos e posteriormente retornadas às câmaras climatizadas. Foram avaliados, com auxílio de microscópio estereoscópico, os seguintes parâmetros: duração (dias) do ciclo de desenvolvimento (ovo-adulto) (realizada através de observações diárias, sempre no mesmo horário), percentual de emergência (efetuada através do

Meira et al., v.10, n.35, p. 10-17, Dourados, 2017 


\section{(ㄱ) \\ Revista Agrarian \\ ISSN: $1984-2538$}

número de ovos com orifício, dividido pelo número de ovos parasitados multiplicado por 100), número de ovos parasitados, número de indivíduos por ovo e razão sexual [calculada a partir da fórmula: $r s=$ $\mathrm{n}^{\mathrm{o}}$ de fêmeas/ $\left(\mathrm{n}^{\mathrm{o}}\right.$ de fêmeas $+\mathrm{n}^{\mathrm{o}}$ de machos $\left.)\right]$. $\mathrm{O}$ sexo dos descendentes foi determinado através do dimorfismo apresentado pelas antenas (fêmeas antenas clavadas e machos antenas plumosas) (Figura 1).
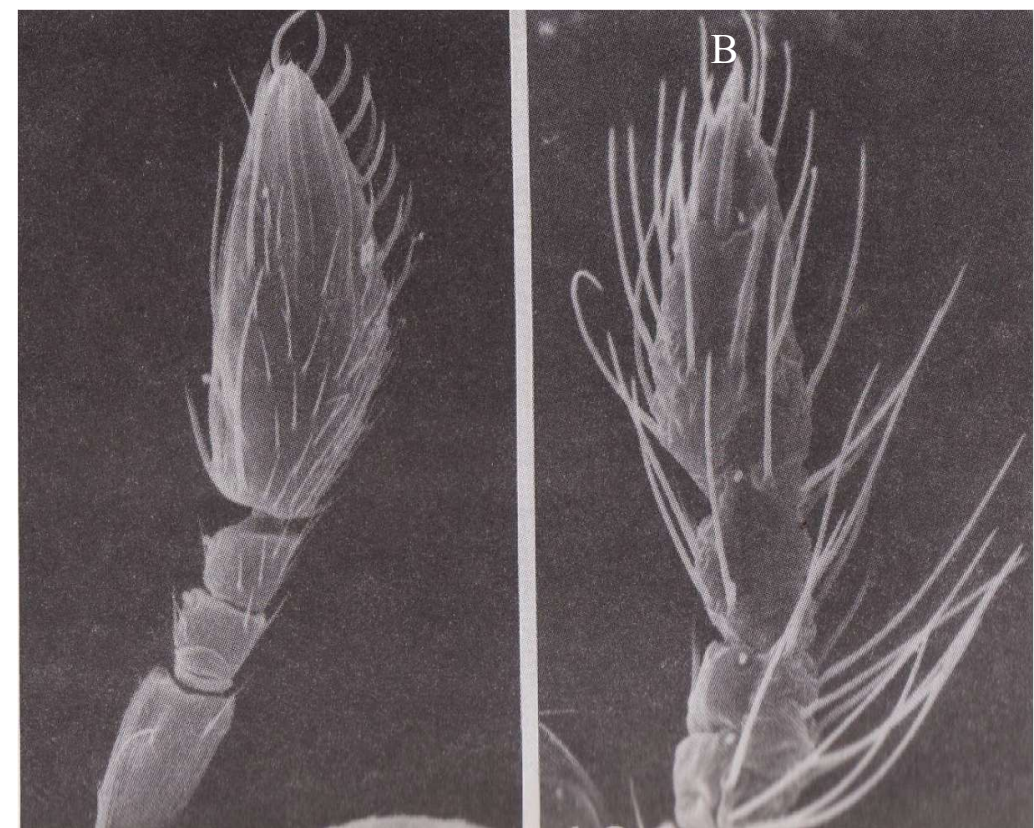

Figura 1. Dimorfismo sexual em espécies de Trichogramma. 1. A antena da fêmea de Trichogramma sp, com clava apical. 1. B antena do macho de Trichogramma sp. (PINTO, 1997).

\section{Análises estatisticas}

O delineamento experimental utilizado foi inteiramente casualizado, com dois tratamentos (espécies de Trichogramma) e dez repetições para cada hospedeiro. Os dados foram submetidos à análise de variância e as médias comparadas pelo teste Tukey, ao nível de $5 \%$ de probabilidade, utilizando-se o programa estatístico SAEG (versão 5.0). Os dados de porcentagem de emergência foram transformados em arco seno $\sqrt{ }(\mathrm{x} / 100)$ e da razão sexual em $\sqrt{ }(x+0,8)$ para atender os prérequisitos da ANOVA.

\section{Resultados e Discussão}

Parâmetros biológicos no periodo de ovo a adulto As espécies de Trichogramma não apresentaram diferença quando criados com os hospedeiros, A. kuehniella, H. zea e P. xylostella para a duração do ciclo de desenvolvimento. Sendo maior quando os ovos destes hospedeiros foram submetidos a T. exiguum e T. atopovirilia emergiu em menor tempo. Não houve diferença para o percentual de emergência entre os três hospedeiros em estudo, totalizando $100 \%$ de emergência (Tabela1).

Os resultados observados por Bueno et al. (2009), ao analisar, duração do período de ovo a adulto, em 13 linhagens de $T$. pretiosum criadas em ovos de Pseudoplusia includens em temperatura de $25 \pm 1{ }^{\circ} \mathrm{C}$, UR de $70 \pm 10 \%$ e fotofase de $14 \mathrm{~h}$ e por Meira et al. (2011), quando espécies de Trichogramma foram criadas no hospedeiro $P$. xylostella, nas mesmas condições de temperatura, umidade e fotofase, corroboram com os resultados obtidos neste trabalho. No entanto, Foresti et al. $(2012)$ obteve resultados que oscilaram entre 8,15 a 8,36, com linhagens de $T$. pretiosum e $T$. atopovirilia, nos hospedeiros $H$. zea e $A$. kuehniella. 


\section{(2) \\ Revista Agrarian \\ ISSN: $1984-2538$}

Tabela 1. Duração do ciclo de desenvolvimento (ovo-adulto) de T. atopovirilia e T. exiguum criadas em ovos de A. kuehniella, H. zea e P. xylostella, a $25 \pm 1{ }^{\circ} \mathrm{C}, 70 \pm 10 \%$ UR e $12 \mathrm{~h}$ de fotofase.

\begin{tabular}{lccc}
\hline & Período de ovo-adulto & & \\
& Duração (dias) & & \\
\hline Espécies de Trichogramma & Anagasta kuehniella & Helicoverpa zea & Plutella xylostella \\
\hline Trichogramma atopovirilia & $9,1 \pm 0,10 \mathrm{Ab}$ & $9,0 \pm 0,00 \mathrm{Ab}$ & $9,2 \pm 0,13 \mathrm{Ab}$ \\
Trichogramma exiguum & $11,0 \pm 0,00 \mathrm{Aa}$ & $11,0 \pm 0,00 \mathrm{Aa}$ & $11,0 \pm 0,00 \mathrm{Aa}$ \\
\hline & Percentual de emergência & \\
\hline Espécies de Trichogramma & Anagasta kuehniella & Helicoverpa zea & Plutella xylostella \\
Trichogramma atopovirilia & $100,0 \pm 0,00 \mathrm{Aa}$ & $100,0 \pm 0,00 \mathrm{Aa}$ & $100,0 \pm 0,00 \mathrm{Aa}$ \\
Trichogramma exiguum & $100,0 \pm 0,00 \mathrm{Aa}$ & $100,0 \pm 0,00 \mathrm{Aa}$ & $100,0 \pm 0,00 \mathrm{Aa}$ \\
\hline
\end{tabular}

Médias ( $\pm \mathrm{EP})$ seguidas de mesma letra, minúscula na linha e maiúscula na coluna, não difere entre si pelo teste Tukey, a $5 \%$ de probabilidade.

Estas diferenças podem estar relacionadas com a qualidade nutricional e o tamanho do ovo, pois este pode conter maior ou menor quantidade de nutrientes, possibilitando o desenvolvimento de um ou mais indivíduos de Trichogramma (Nava et al., 2007). A variação de espécies de Trichogramma, para duração do período de ovo a adulto, indica que este parâmetro biológico depende da temperatura, do hospedeiro e da cultura em que foi coletado. Podendo ser confirmado por Altoé et al. (2012), ao estudar parasitismo de $T$. pretiosum criados em ovos de Trichoplusia $n i$ (Lepidoptera: Noctuidae) em diferentes temperaturas.

Para o percentual de emergência, resultados semelhantes foram verificados para $T$. exiguum quando criados em ovos de Trichoplusia ni Hübner (Lepidoptera: Noctuidae) e T. pretiosum submetidos a ovos de diferentes hospedeiros (Volpe et al., 2006; Milanez et al., 2009). Dias-Pini et al. (2012) verificaram o percentual de emergência de $82 \%$, quando ovos de Diatraea flavipennella (Lepidoptera, Crambidae) foram utilizados como hospedeiros para Trichogramma galloi. Deste modo, devido ao elevado percentual de emergência das espécies de Trichogramma do presente estudo, a interação com as espécies $A$. kuehniella, $P$. xylostella e H. zea foi considerada como adequada.

\section{Parâmetros biológicos no estágio adulto}

Os parâmetros biológicos avaliados no estágio adulto, para número de ovos parasitados, somente A. kuehniella, diferenciou entre as espécies de Trichogramma em estudo quando comparados com H. zea e P. xylostella. Para o número de indivíduos por ovo, os maiores valores foram observados quando $H$. zea foi designada como hospedeira de $T$. atopovirilia e de $T$. exiguum. A razão sexual, $T$. atopovirilia diferiu apenas para $A$. kuehniella quando comparado com os demais hospedeiros e entre as espécies de Trichogramma em estudo (Tabela 2).

Em relação ao número de ovos parasitados, T. atopovirilia parasitou 14,8 ovos e $T$. exiguum 14,4 , sendo o maior número de ovos parasitados quando comparado com os demais hospedeiros e H. zea diferiu estatisticamente entre as espécies de Trichogramma. Diferenças no número de ovos parasitados têm sido relatadas em estudos fornecendo uma boa indicação para espécies de Trichogramma no controle de $P$. xylostella (Lepidoptera: Crambidae) e Diaphania hyalinata (Meira et al., 2011; Polanczyk et al., 2012).

Magalhães et al. (2012), ao estudar dados biológicos de espécies de Trichogramma em $A$. kuehniella e $P$. xylostella, utilizando diferentes cores de cartelas, obteve resultados semelhantes ao usar cartela de cor azul, mesma cor utilizada neste trabalho, para número de ovos parasitados quando criado em $P$. xylostella. E maior número de ovos parasitados, quando foram oferecidos os ovos de $A$. kuehniella para a espécie de Trichogramma, corroborando com os resultados apresentados neste trabalho. 


\section{(ㅇ)}

\section{Revista Agrarian}

ISSN: 1984-2538

Tabela 2. Parâmetros biológicos avaliados no estágio adulto para T. atopovirilia e T. exiguum criadas em ovos de A. kuehniella, H. zea e P.xylostella, a $25 \pm 1^{\circ} \mathrm{C}, 70 \pm 10 \%$ UR e $12 \mathrm{~h}$ de fotofase.

Estágio adulto

\begin{tabular}{|c|c|c|c|}
\hline \multicolumn{4}{|c|}{ Número de ovos parasitados } \\
\hline Espécies de Trichogramma & Anagasta kuehniella & Helicoverpa zea & Plutella xylostella \\
\hline Trichogramma atopovirilia & $14,8 \pm 1,33 \mathrm{Aa}$ & $9,3 \pm 1,35 \mathrm{Bb}$ & $12,1 \pm 1,29 \mathrm{Ba}$ \\
\hline Trichogramma exiguum & $14,4 \pm 1,15 \mathrm{Aa}$ & $11,2 \pm 0,70 \mathrm{Ba}$ & $11,6 \pm 1,03 \mathrm{Ba}$ \\
\hline \multicolumn{4}{|c|}{ Número de indivíduos por ovo } \\
\hline Espécies de Trichogramma & Anagasta kuehniella & Helicoverpa zea & Plutella xylostella \\
\hline Trichogramma atopovirilia & $1,0 \pm 0,04 \mathrm{Ba}$ & $1,3 \pm 0,10 \mathrm{Ab}$ & $1,0 \pm 0,01 \mathrm{Ba}$ \\
\hline Trichogramma exiguum & $1,1 \pm 0,09 \mathrm{Ba}$ & $1,9 \pm 0,12 \mathrm{Aa}$ & $1,0 \pm 0,02 \mathrm{Ba}$ \\
\hline \multicolumn{4}{|c|}{ Razão sexual } \\
\hline Espécies de Trichogramma & Anagasta kuehniella & Helicoverpa zea & Plutella xylostella \\
\hline Trichogramma atopovirilia & $0,7 \pm 0,04 \mathrm{Bb}$ & $0,8 \pm 0,04 \mathrm{Ab}$ & $0,8 \pm 0,04 \mathrm{Ab}$ \\
\hline Trichogramma exiguum & $1,0 \pm 0,00 \mathrm{Aa}$ & $1,0 \pm 0,00 \mathrm{Aa}$ & $1,0 \pm 0,00 \mathrm{Aa}$ \\
\hline
\end{tabular}

Médias ( $\pm \mathrm{EP})$ seguidas de mesma letra, minúscula na linha e maiúscula na coluna, não difere entre si pelo teste Tukey, a $5 \%$ de probabilidade.

O maior número de ovos parasitados obtido ao utilizar ovos de $A$. kuehniella pode estar relacionado com a aceitação do hospedeiro em relação à herança genética e as características herdadas em várias gerações (Goulart et al., 2008), pois para a manutenção das espécies de Trichogramma são oferecidos ovos de $A$. kuehniella.

Para o número de indivíduos por ovo, os maiores valores foram observados quando $H$. zea foi designada como hospedeira de T. atopovirilia e de T. exiguum. O maior número de indivíduos por ovo foi observado quando H. zea foi utilizada como espécie hospedeira de $T$. exiguum. Resultados equivalentes foram obtidos em estudos de parasitismo de espécies de Trichogramma em diferentes hospedeiros (Nava et al., 2007; Milanez et al., 2009, Bellon et al., 2014). No entanto Parra \& Zachrisson (2011), obtiveram resultados superiores, ao submeter Trichogramma pretiosum em ovos de Anticarsia gemmatalis para diferentes temperaturas.

A razão pela qual o maior número de indivíduos terem sido obtidos quando em ovos do hospedeiro $H$. zea neste trabalho, é devido ao tamanho do ovo. A emergência de mais de um indivíduo por ovo foi relacionada ao maior volume do ovo do hospedeiro, devido à maior quantidade de nutrientes, elevando a disponibilidade de recursos e garantindo a geração de um maior número de indivíduos mais fortes e competitivos (Nava et al., 2007).

Em relação razão sexual, resultados próximos foram encontrados por Milanez et al.
(2009), ao utilizar as mesmas espécies de Trichogramma em Trichoplusia ni, por Meira et al. (2011) ao utilizar espécies de Trichogramma em $P$. xylostella ao parasitismo no período de $24 \mathrm{~h}$ e ao parasitismo diário durante a fase adulta. Entretanto, estudo realizado por Melo et al. (2007) em $T$. atopovirilia, submetidos a ovos de Diaphania hyalinata Linnaeus (Lepidoptera: Crambidae) em mesma temperatura e umidade relativa, porém com $14 \mathrm{~h}$ de fotofase, a razão sexual foi inferior aos resultados obtidos neste ensaio.

A razão sexual foi alta para as espécies utilizadas, pois o nascimento de maior quantidade de fêmeas é desejável em programas de controle biológico, porque quanto maior o número de fêmeas maior o potencial de controle (Wakeil et al., 2008).

Em relação aos parâmetros avaliados, as duas espécies de Trichogramma estão aptas a se desenvolverem nos hospedeiros A. kuehniella e $P$. xylostella, pois não houve diferença em relação ao número de ovos parasitados, percentual de emergência e número de individuo por ovo. Essas variações entre espécies de Trichogramma, principalmente no parasitismo, mostram a importância de se avaliar o maior número de espécies e linhagens desse parasitóide.

Em relação ao hospedeiro $H$. zea, $T$. exiguum foi à espécie que obteve melhor desempenho, parasitando maior número de ovos e obtendo maior número de indivíduos por ovo. Provavelmente se os ovos fornecidos à espécie de Trichogramma tivessem menos de 24 horas de desenvolvimento embrionário, seriam

Meira et al., v.10, n.35, p. 10-17, Dourados, 2017 


\section{(-) \\ Revista Agrarian \\ ISSN: 1984-2538}

considerados mais atrativos, pelo elevado teor de nutrientes favorável para o desenvolvimento dos descendentes destes parasitóides.

\section{Conclusão}

Potencialmente as duas espécies de Trichogramma poderão ser utilizadas através de liberações inundativas associadas a outros métodos de controle dentro de um programa de manejo fitossanitário, tais como resistência de plantas e uso de produtos químicos seletivos, nos respectivos hospedeiros.

Agradecimentos: À Coordenação de Aperfeiçoamento de Pessoal de Ensino Superior CAPES, pela bolsa de estudo concedida ao primeiro autor.

\section{Referências}

ALTOÉ, T. S.; PRATISSOLI, D.; CARVALHO, J. R.; SANTOS JÚNIOR, H. J. G.; PAES, J. P. P. BUENO, R. C. O. F.; BUENO, A. Trichogramma pretiosum (Hymenoptera: Trichogrammatidae) parasitism of Trichoplusia ni (Lepidoptera: Noctuidae) eggs under different temperatures. Annals of the Entomological Society of America, v.105, n.1, p.82-89, 2012.

BELLON, P. P.; SANTANA, D. R. S.; GLAESER, D. F.; QUERINO, R. B.; FELTRIN, E.; OLIVEIRA, H. N. Biological characteristics of Trichogramma pretiosum (Hymenoptera: Trichogrammatidae) in Anagasta kuehniella (Lepidoptera: Pyralidae) and Diatraea saccharalis (Lepidoptera: Crambidae). Cadernos de Agroecologia, v.9, n. 4, p. 1-8, 2014.

BOIÇA JÚNIOR, A. L.; JANINI, J. C.; SOUZA, B. H. S. de; RODRIGUES, N. E. L. Efeito de cultivares de repolho e doses de extrato aquoso de nim na alimentação e biologia de Plutella xylostella (Linnaeus) (Lepidoptera: Plutellidae). Bioscience Journal, v. 29, n. 1, p. 22-31, 2013.

BOIÇA JUNIOR, A. L.; TAGLIARI, S. R. A.; PITTA, R. M.; JESUS, F. G. de; BRAZ, L. T. Influência de genótipos de couve (Brassica oleracea L. var. acephala DC.) na biologia de Plutella xylostella (L., 1758) (Lepidoptera: Plutellidae). Ciência e Agrotecnologia, Lavras, v. 35, n. 4, p. 710-717, 2011.

BUENO, R. C. O. F.; PARRA, J. R. P.; BUENO, A. F.; HADDAD, M. L. Desempenho de tricogramatídeos como potenciais agentes de controle de Pseudoplusia includens Walker (Lepidoptera: $\quad$ Noctuidae). Neotropical Entomology, v.38, n.3, p.389-394, 2009.

CARDOSO, M. O.; BERNI, R. F.; KRUG, C.; ANTONIO, I. C. Danos por Plutella xylostella em couve de folhas jovem afetados pela altura e pelo nitrogênio. Manaus: Embrapa Amazônia Ocidental, 2012, 19p.

DIAS-PINI, N. S.; BROGLIO, S. M. F.; COSTA, S. S.; SANTOS, J. M. S.; GUZZO, E. C. Biological characteristics of Telenomus alecto and Trichogramma galloi reared on eggs of the sugarcane borer Diatraea flavipennella. Revista Brasileira de Entomologia, v.56, n.4, p.515-518, 2012.

FORESTI, J.; GARCIA, M. S.; BERNARDI, O.; ZART, M. ; NUNES, A. M. Biologia, Seleção e Avaliação de Linhagens de Trichogramma spp. para o Controle da Lagarta-da-Espiga em Milho Semente. EntomoBrasilis, v.5, n. 1, p.43-4, 2012.

FREITAS, J. R.; SILVA, A. B.; BESERRA, E. B.; DANTAS, J. P. Flutuação populacional e parasitismos de ovos de Spodoptera frugiperda e Helicoverpa zea em milho pulverizado com extratos vegetais e Metarhizium anisopliae. Engenharia Ambiental, v. 7, n. 3, p. 291-300, 2010.

GOULART, R.M.; DE BORTOLI, S.A.; THULER, R.T.; PRATISSOLI, D.; VIANNA, C.L.T.P.; VOLPE, H.X.L. Avaliação da seletividade de inseticidas a Trichogramma spp. (Hymenoptera: Trichogrammatidae) em diferentes hospedeiros. Arquivos do Instituto Biológico, v.75, n.1, p.69-77, 2008.

MAGALHÃES, G. O.; GOULART, R. M.; VACARI, A. M.; BORTOLI, S. A. Parasitismo de Trichogramma pretiosum Riley, 1879 (Hymenoptera: Trichogrammatidae) em hospedeiros e cores de cartelas. Arquivo do Instituto Biológico, v.79, n.1, p.55-60, 2012

MEIRA, A. L.; PRATISSOLI, D.; SOUZA, L. P.; STURM, G. Seleção de espécies de Trichogramma sp. em ovos da traça das crucíferas, Plutella xylostella. Revista Caatinga, v.24, n. 2, p.1-8, 2011.

MELO, R.; PRATISSOLI, D.; POLANCZYK, R.; MELO, D.; BARROS, R.; MILANEZ, A. Biologia 


\section{(a) \\ Revista Agrarian \\ ISSN: 1984-2538}

e exigências térmicas de Trichogramma atopovirilia Oatman \& Platner (Hymenoptera: Trichogrammatidae) em ovos de Diaphania hyalinata L. (Lepidoptera: Pyralidae). Neotropical Entomology, v. 36, n.3, p. 431-435, 2007.

MILANEZ, A. M.; PRATISSOLI D.; POLANCZYK, R. A.; BUENO, A. F.; TUFK, C. B. A. Avaliação de Trichogramma spp. para o controle de Trichoplusia ni. Pesquisa Agropecuária Brasileira, v. 44, n. 10, p 12191224, 2009.

NAVA, D. E; TAKAHASHI, K. M; PARRA, J. R. P. Linhagens de Trichogramma e Trichogrammatoidea para controle de Stenoma catenifer. Pesquisa Agropecuária Brasileira, v. 42, n. 1, p 9-16, 2007.

PEREIRA, F. F.; BARROS, R.; PRATISSOLI, D.; PEREIRA, C. L. T.; VIANNA, U. R.; ZANUNCIO, J. C. Capacidade de parasitismo durante a fase adulta de Trichogramma exiguum Pinto \& Platner, 1978 (Hymenoptera: Trichogrammatidae) em ovos de Plutella xylostella (Lepidoptera: Plutellidae) em diferentes temperaturas. Ciência Rural, v. 37, n.2, p. 297303, 2007.

PINTO, J.D. Taxonomia de Trichogramma. In: PARRA, J.R.P. \& ZUCCHI, R.A. Trichogramma e o controle biológico aplicado. Piracicaba: FEALQ, 1997. p. 19-40.

POLANCZYK, R. A.; BARBOSA, W. F.; CELESTINO, F. N.; PRATISSOLI, D.; HOLTZ, A. M.; MILANEZ, A. M.; COCHETO, J. G.; SILVA, A. F. Influência da densidade de ovos de Diaphania hyalinata (L.) (Lepidoptera: Crambidae) na capacidade de parasitismo de Trichogramma exiguum Pinto \& Platner e Trichogramma pretiosum Riley (Hymenoptera: Trichogrammatidae). Neotropical Entomology, v. 40, n.2, p. 238-243, 2011.

PRATISSOLI, D.; POLANCZYK, R. A.; HOLTZ, A. M.; DALVI, L. P.; SILVA, A. F.; SILVA, L. N. Selection of Trichogramma species for controlling the diamondback moth. Horticultura Brasileira, v. 26, n. 2, p. 194-196, 2008.

SAEG. Sistema para Análise Estatística e Genética v. 5.0. Viçosa, UFV, 1997, 150p.

VIANNA, U. R.; PRATISSOLI, D.; ZANUNCIO, J. C.; ALENCAR, J. R. C. C.; ZINGER, F. D.
Espécies e/ou linhagens de Trichogramma spp. (Hymenoptera: Trichogrammatidae) para o controle de Anticarsia germatalis (Lepidoptera: Noctuidae). Arquivo do Instituto Biológico, v.78, n.1, p.81-87, 2011.

VOLPE, H. X. L.; BORTOLI, S. A.; THULER, R. T.; VIANNA, C. L. T. P.; GOULART, R. M. Avaliação de características biológicas de Trichogramma pretiosum Riley (Hymenoptera: Trichogrammatidae) criado em três hospedeiros. Arquivo do Instituto Biológico, v.73, n. 3, p. 311315, 2006.

WAKEIL, N.E.; FARGHALY, H.T.; RAGAB, Z.A. Efficacy of inundative releases of Trichogramma evanescens in controlling Lobesia botrana in vineyards in Egypt. Journal of Pesticide Science, v.81, n.1, p.49-55, 2008.

WANG X; LI X; SHEN A; WU Y. Baseline susceptibility of the diamondback moth (Lepidoptera: Plutellidae) to chlorantraniliprole in China. Journal of Economic Entomology, v.103, n. 3, p.843-848, 2010.

ZACHRISSON, B.; PARRA, J. R. P. Biología de Trichogramma pretiosum Riley, 1879 (Himenoptera: Trichogrammatidae), parasitoide oófago de Anticarsia gemmatalis Hübner, 1818 (Lepidoptera, Noctuidae), en diferentes temperaturas. Tecnociencia, v. 13, n. 2, p.33-45, 2011. 